\title{
Treatment planning study of the 3D dosimetric differences between Co-60 and Ir-192 sources in high dose rate (HDR) brachytherapy for cervix cancer
}

\author{
Antony Palmer, MSc, BSc'², Orla Hayman, MSc, BSc', Sarah Muscat, PhD, MSc, BSc' \\ IMedical Physics Department, Queen Alexandra Hospital, Portsmouth Hospitals NHS Trust, Portsmouth, PO6 3LY, UK, 2Department of Physics, \\ Faculty of Engineering and Physical Science, University of Surrey, Guildford, GU2 7XH, UK
}

\begin{abstract}
Purpose: To evaluate whether Co-60 is equivalent to Ir-192 for HDR cervical brachytherapy, through 3D-DVH dose comparisons in standard and optimised plans. Previous studies have only considered 2D dosimetry, point dose comparisons or identical loading. Typical treatment times and economics are considered.

Material and methods: Plans were produced for eight cervix patients using Co-60 and Ir-192 sources, CT imaging and IU/two-channel-ring applicator (Eckert Ziegler BEBIG). The comparison was made under two conditions: (A) identical dwell positions and loading, prescribed to Point A and (B) optimised source dwells, prescribed to HR-CTV. This provided a direct comparison of inherent differences and residual differences under typical clinical plan optimisation. The DVH (target and OAR), ICRU reference points and isodose distributions were compared. Typical treatment times and source replacement costs were compared.

Results: Small differences $(p<0.01)$ in 3D dosimetry exist when using Co-60 compared to Ir-192, prescribed to Point A with identical loading patterns, particularly 3.3\% increase in rectum D2cc. No significant difference was observed in this parameter when prescribing to the HR-CTV using dwell-time optimisation. There was no statistically significant difference in D90 between the two isotopes. Co-60 plans delivered consistently higher V150\% (mean $+4.4 \%, p=0.03$ ) and V400\% $($ mean $+11.6 \%, p<0.01)$ compared to Ir-192 in optimised plans. Differences in physical source properties were overwhelmed by geometric effects.

Conclusions: Co-60 may be used as an effective alternative to Ir-192 for HDR cervix brachytherapy, producing similar plans of equivalent D90, but with logistical benefits. There is a small dose increase along the extension of the source axis when using Co-60 compared to Ir-192, leading to small rectal dose increases for identical loading patterns. This can be eliminated by planning optimisation techniques. Such optimisation may also be associated with increases in the overdose volume (V150-V400) with Co-60 compared to Ir-192.
\end{abstract}

J Contemp Brachyther 2012; 4, 1: $52-59$ DOI: $10.5114 / j \mathrm{jcb} .2012 .27952$

Key words: high dose-rate (HDR), brachytherapy, cervix cancer, Co-60, treatment plan.

\section{Purpose}

There are currently in excess of 150 radiotherapy centres worldwide using Co-60 sources in modern high dose-rate (HDR) brachytherapy treatment units. These new systems utilise miniaturised Co-60 sources, rather than traditional Ir-192 sources and becoming very popular due to longer source replacement intervals, lower operating costs and a reduced frequency of movement of radioactive sources between countries, compared to Ir-192 [1]. Whilst the availability of miniaturised high specific-activity Co-60 sources for high dose-rate brachytherapy is a recent development, the use of physically larger Co-60 sources in low dose-rate applications has a long history dating back to the 1960s and 1970s with the Cathetron, Ralstron and Selectron treatment units, reported in 1964 by Henschke [2]. By the 1980s, Ir-192 had become the most popular isotope for HDR brachytherapy due to its smaller physical size. The application of HDR brachytherapy in carcinoma of the cervix is a well established treatment technique [3] and work by Dale [4], has shown there is negligible clinical significance in the biological response of tissues of varying density to the different energy spectra from Co-60 and Ir-192.

There are several publications on the clinical use of Co- 60 HDR and on comparisons of physical dose differences with 
much more common Ir-192 sources. Ntekim et al. [5], Mosalaei et al. [6] and Bocharova [7] have evaluated acute toxicity with Co-60 in gynaecological cancer and shown this to be a tolerable isotope for HDR treatments, reporting the results to be comparable to Ir-192. The dose deposition differences around single Co-60 and Ir-192 sources (anisotropy, radial dose function and isodose curves) have been reviewed by Strohmaier [8], finding no advantage or disadvantage for Co-60 sources compared to Ir-192. However, this review is based on the available work by Venselaar [9], Richter et al. [10] and Park et al. [11], who confined their analysis to point dose and qualitative isodose comparisons only. Park et al. [11] considered cervix treatment plans and compared only the ICRU reference point doses [12] for two Ir-192 sources and a Co- 60 source using treatment plans based on 2D orthogonal radiographs, finding rectal doses to be higher (average $+0.8 \%$ ) and bladder doses lower (average -1.1\%) for Co-60 compared to Ir-192. Richter et al. [10] and Park et al. [11] have also only considered differences in dose distributions produced by treatment plans with identical source dwell loading patterns. While this provides valuable information on the inherent physical differences between the sources, what is also required is information on any residual differences in typical clinical treatment plans, where non-identical dwell-times are allowed with the two isotopes; i.e. comparing actual typical treatment plans that may be achieved with Co-60 and Ir-192. Whilst prescription to 'Manchester system' Point A is still common, an evaluation of the differences between the two isotopes under the improved dosimetry recommendations from GEC-ESTRO [13] is required: based on $3 \mathrm{D}$ treatment planning and $\mathrm{DVH}$ parameter reporting, in order to fully and comprehensively evaluate differences in treatment plan dose distributions between Co-60 and Ir-192 sources for HDR gynaecological brachytherapy.

The current work builds on previous comparisons of physical characteristics of the two isotopes and simple planning comparisons, by investigating Co-60 and Ir-192 brachytherapy treatment plans in terms of dose-volume histogram (DVH) reporting metrics, with both standard identical loading patterns prescribed to Point A and optimised plans prescribed to the high-risk clinical target volume (HR-CTV). Typical treatment times and source replacement costs are also investigated.

\section{Material and methods}

The comparison between Co-60 and Ir-192 isotopes was undertaken by considering the source types manufactured by Eckert \& Ziegler BEBIG GmbH, Germany (in future termed "EZ BEBIG"), models Co0.A86 and Ir.A85-2, respectively. These sources are geometrically identical and were therefore ideal to compare the effects of isotope choice. Both sources consist of an active source core of length $3.5 \mathrm{~mm}$ and diameter $0.6 \mathrm{~mm}$, surrounded by a cylindrical steel jacket with a length of $5 \mathrm{~mm}$ and an outer diameter of $1.0 \mathrm{~mm}$. Monte Carlo derived dosimetric parameters for the two source types were utilised [14-16], alongside published data on the physical properties of the two isotopes.

\section{Treatment plans}

A consecutive series of eight cervix cancer patients were planned for HDR brachytherapy using the "HDRPlus ${ }^{\circledR}$ " treatment planning system (version 2.5), supplied by EZ BEBIG, on CT image data using TG43 [17] calculation algorithm. Pujades-Claumarchirant et al. have shown the TG43 algorithm approach will provide accuracy within $2 \%$ for both Co-60 and Ir-192 sources [18]. A diagnostic MRI scan acquired post-external beam radiotherapy was additionally available in order to improve soft tissue outlining, although this was not acquired with applicators in situ and not directly fused with the $\mathrm{CT}$ data set. An experienced Consultant Clinical Oncologist outlined HR-CTV and organs at risk (OAR): rectum, sigmoid and bladder on each data set. Given this is a planning comparison, it was not considered necessary to have these volumes independently reviewed.

The EZ BEBIG GmbH "CT/MR Ring Applicator Set", codes SET0140-3, was used in all treatments. This applicator consists of an intrauterine tube (IU) and a two-channelring. The possible source dwell positions are physically similar to a Fletcher-style IU and ovoids with the lateral straight channels being physically housed within a plastic ring of outer diameters 36 to $45 \mathrm{~mm}$. Dwell positions within the EZ BEBIG "ring" are actually in a straight line rather than curved geometry, as would be anticipated from the physical ring appearance. The planning system libraryapplicators were used in all treatment plans and the applicators were not moved between the Co-60 and Ir-192 planning for each patient, to eliminate any uncertainty due to applicator reconstruction.

Standard loading patterns (positions and times of source dwells within the applicators) based on the traditional Manchester system were utilised, with relative total dwell-time loadings of IU and ring of typically $1.25: 1$ (ranging between $1: 1$ and $1.56: 1$, depending on the length of IU and diameter of ring combination), producing typical 'pear-shaped' isodose curves. Supplementary interstitial needles were not used in any of the plans. Treatment plans were produced for each patient using Co-60 source and then again using Ir-192 source in two conditions: (A) traditional approach with fixed dwell distribution normalised to $100 \%$ of prescription dose at Point A [12] and (B) optimised dwell patterns prescribed to HR-CTV [13] with manual adjustment of dwell times, but using the same dwell positions and no inverse planning. In the first set of comparisons, plans were produced with identical dwell positions and relative times (scaled for dose-rate difference) for the two isotopes, to provide a direct comparison of the dose deposition between Co-60 and Ir-192 sources, due to inherent differences in the physical properties between the two sources. In the second comparison set, normal treatment planning approaches were applied to evaluate any residual differences in dosimetry in normal clinical use, i.e. optimisation of dwell times and distributions were applied to reduce dose to the rectum (aim for ICRU rectal reference point $<67 \%$ of prescription dose) using the "local isodose shaping" software function available within the EZ BEBIG HDRplus brachytherapy treatment planning systems. The level of optimisation may unintentionally have been different between plans produced 


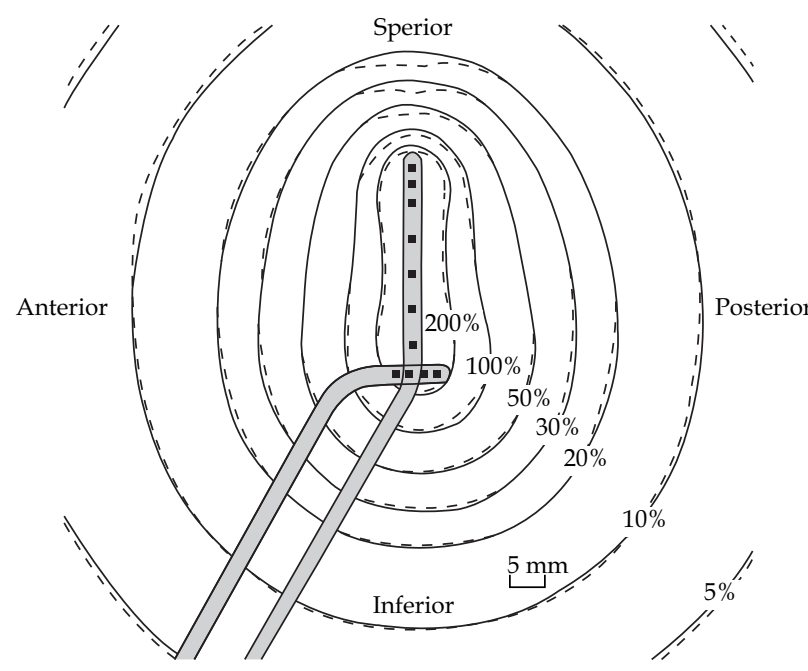

Fig. 1. Comparison of isodose lines $(5,10,20,30,50,100$ and $200 \%$ of prescription dose) for Co-60 source (solid lines) and Ir-192 source (dashed lines) for a standard HDR cervix treatment, with identical dwell positions and relative times, based on Manchester loading, normalised to $100 \%$ of prescription dose at Point A, shown in sagittal projection. (Condition [A] in method)

with the two isotopes to achieve the desired treatment planning aims: coverage of HR-CTV and minimised dose to OAR. To mitigate against this, a single experienced treatment planner produced all treatment plans to ensure the level of plan optimisation and all other parameters were identical between the plans. All patients were actually treated with an optimised Co- 60 plan; the work presented here is a retrospective planning study on the comparison of Co-60 and Ir-192 source delivery.

The following quantitative dose-volume parameters were calculated for each plan: HR-CTV D90 (the dose to $90 \%$ of the HR-CTV), V100\% (the HR-CTV volume covered by the prescription dose), V150\%, V200\% and V400\% (the HR-CTV volumes covered by the stated percentage of prescription dose), and D2cc (maximum dose to $2 \mathrm{~cm}^{3}$ ) for each OAR. The ICRU reference point doses to OARs and Point A were also calculated. A qualitative visual assessment of the variation in isodose line positions was also undertaken. The data was further analysed by comparing a sparing factor, evaluated as the ratio of D2cc for each OAR and D90 for the HR-CTV, to make an assessment of the clinical quality of the plan. Where appropriate, a paired two-sided t-test was used to assess the statistical significance of the differences between the Co-60 and Ir-192 plans.

\section{Technical and economic considerations}

The physical source parameters that relate to the clinical use of Co-60 and Ir-192 HDR sources were compiled. This data was used to evaluate the equivalent source strength of Co-60 compared to Ir-192 to deliver the same dose, the variation in clinical treatment irradiation times to be expected when using the two isotopes and the recommended source change frequencies. The current cost of
Co-60 and Ir-192 sources from EZ BEBIG were also obtained to evaluate the differing cumulative source replacement costs of using the two isotopes for HDR brachytherapy.

\section{Results}

\section{Treatment plans}

Figure 1 illustrates a simple comparison of the isodose lines calculated when using Co-60 and Ir-192 sources for a typical HDR cervix treatment, with EZ BEBIG two-channel split ring and IU applicator, with identical loading for the two sources, prescribed to Point A (condition [A] in method). The figure shows the effect of the inherent differences in the two isotopes on the resulting dose distributions. Differentiation between the plans was observed in isodose shifts of up to $4.0 \mathrm{~mm}$ superior to the intrauterine applicator and up to $2.0 \mathrm{~mm}$ posterior to the ring applicator, both with Co-60 isodoses further from the applicator than the equivalent Ir-192 lines. Similar dose enhancements were observed along the applicator axes inferior and posterior, although of smaller magnitude. In all other regions the isodose lines for Ir-192 were found to be further from the applicator than Co-60 lines, by several $\mathrm{mm}$, with divergence increasing with escalating distance from the applicators; average of $1.0 \mathrm{~mm}$ at $30 \%$ isodose, approximately $20 \mathrm{~mm}$ to $30 \mathrm{~mm}$ from the applicator and $3.0 \mathrm{~mm}$ at $10 \%$ isodose level, approximately $40 \mathrm{~mm}$ to $50 \mathrm{~mm}$ from the applicator (from Fig. 2, discussed later, it can be seen that the radial dose function, $\mathrm{g}(\mathrm{r})$, is larger for Ir-192 than for Co-60 in these regions).

As a result of these differences, in all treatment plans considered, Co-60 plan produced higher-dose lobes along the extension of the applicator axes, delivering locally up to $10 \%$ greater dose within the rectum compared to Ir-192 plan. Consistently lower doses were delivered from Co-60 plans to regions more distant from the applicators, including distant portions of the bladder, rectum outside the high-dose lobe and sigmoid, by up to $1.5 \%$ compared to Ir- 192 plans.

Quantitative analysis of the difference in point-dose (ICRU [12]) and dose-volume parameters (GEC-ESTRO [13]) between Co-60 and Ir-192 plans is given in Table 1: (A) for plans prescribed to Point A with identical loading and (B) prescribed to HR-CTV with clinically optimised loading. In all plans, optimisation of the dwell times reduces the dose differences apparent between Co-60 and Ir-192 plans in the identical loading case.

For the plans prescribed to Point A with identical loading, Table 1 (A) there were statistically significantly differences $(p<0.01)$ in all of the volume parameters for HR-CTV between Co-60 and Ir-192 plans, with Co-60 delivering higher doses in each case, with a mean of $2.4 \%$ for $\mathrm{V} 100 \%$, $5.9 \%$ for $\mathrm{V} 150$, increasing to $22.1 \%$ for $\mathrm{V} 400 \%$. There was no statistically significant difference for the HR-CTV D90Gy. There was also no significant difference in the bladder D2cc or ICRU bladder reference point nor the sigmoid D2cc, between Co-60 and Ir-192 plans. However, the rectum D2cc and ICRU rectal reference point both show statistically significant increased doses from Co-60 compared to Ir-192 plans $(+3.3 \%(p<0.01)$ and $+2.2 \%(p=0.03)$, respectively). 


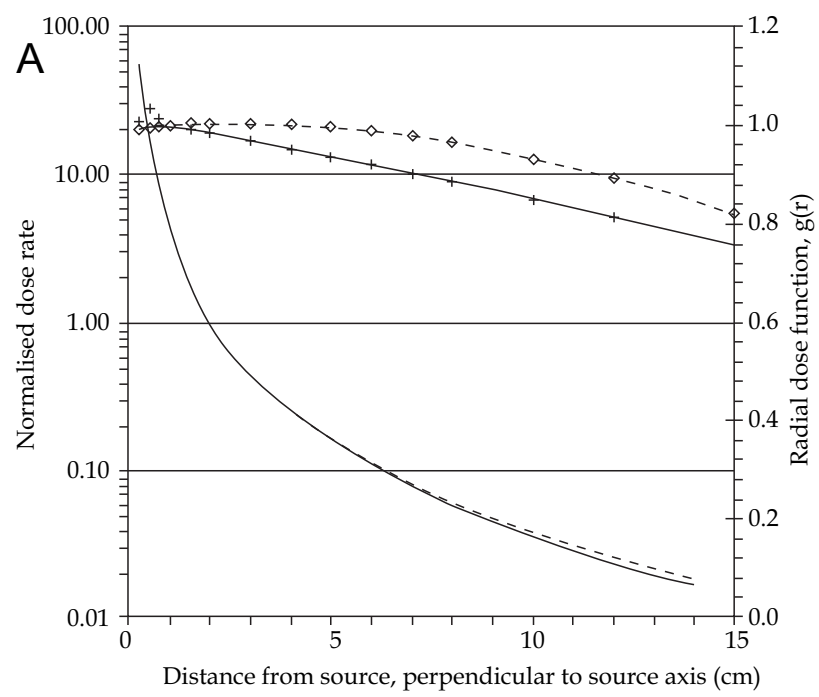

$\begin{array}{ll}- \text { Co-60 dose rate } & -- \text { - Ir-192 dose rate } \\ \longrightarrow \text { Radial dose function Co-60 } & \diamond-\text { Radial dose function Ir-192 }\end{array}$

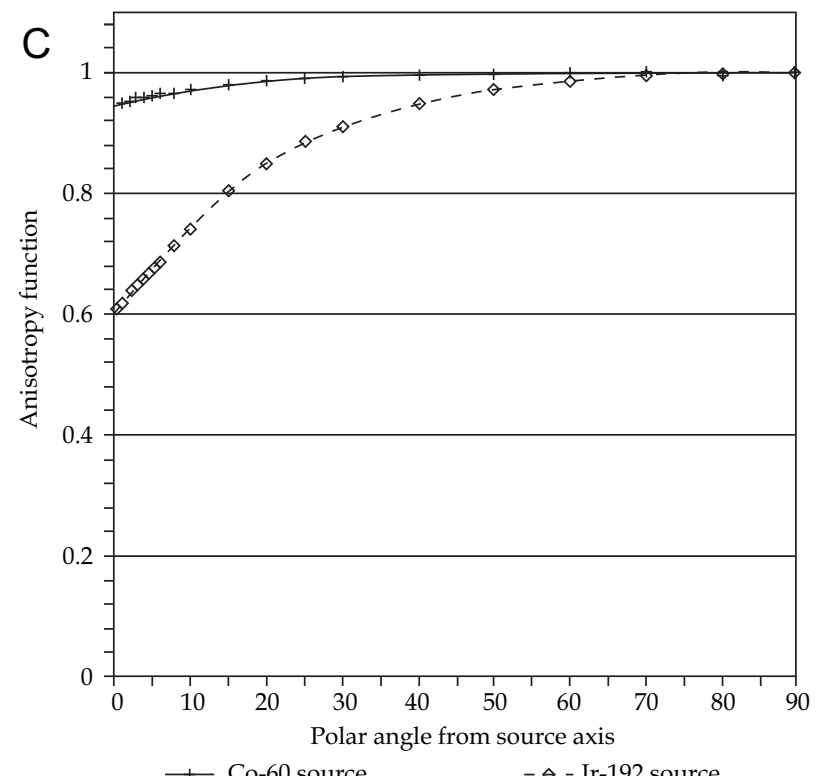

For the plans prescribed to the HR-CTV with clinically optimised loading, Table 1 (B) there was no significant difference in the HR-CTV V100\% and D90Gy nor any OAR $\mathrm{D} 2 \mathrm{cc}$. There was significant difference in the higher-percentage dose volume parameters, with Co-60 delivering larger dose than Ir-192 for by $4.4 \%$ at V150\%, increasing to $11.6 \%$ at $\mathrm{V} 400 \%$.

Figure 3 presents the bladder, rectum and sigmoid sparing factors (evaluated as D2cc/D90) for Co-60 and Ir-192 plans for the eight patients, with all plans prescribed to HR-CTV with clinical dwell-time optimisation (condition [B] in method). The p-value of a paired t-test was just below statistical significance for a comparison of Co-60 and Ir-192 sparing factors for the rectum $(p=0.058)$, with means of 0.88 and 0.85 , respectively. There was a statistically sig-

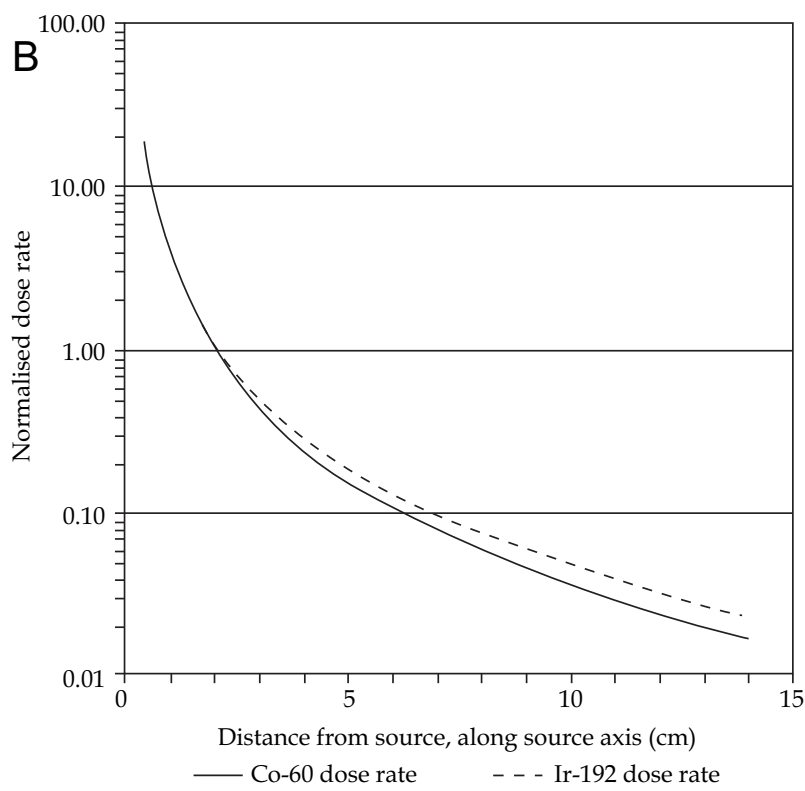

Fig. 2. Dose distribution around the Ir-192 and Co-60 sources in a uniform unit-density medium. Dose rate variation as a function of distance from the sources, normalised at $2.0 \mathrm{~cm},(\mathrm{~A})$ perpendicular to the source axis and (B) along the source axis. The radial dose functions are also shown in (A). (C) Anisotropy function variation with polar angle around the sources, at $2.0 \mathrm{~cm}$ from the source centre, with 0 degrees at the distal source tip

nificant difference for the sigmoid sparing factor $(p=0.05)$ but with a small magnitude difference in the mean values, 0.62 and 0.63 for Co-60 and Ir-192, respectively. There was no statistically significant difference for the bladder $(p=0.51)$. Care must be taken in interpretation of this statistical analysis due to the relatively small sample size $(n=8)$.

\section{Technical and economic considerations}

Table 2 provides a compilation of the physical source parameters relevant to the clinical use of Co-60 and Ir-192 HDR sources. The equivalent source strength of Co-60 to deliver the same dose as Ir-192 was evaluated using simple calculations with the above data: according to the TG43 formulism [17], the absorbed dose is proportional to the source 
Table 1. Comparison of point and dose-volume parameters for the treatment plans of eight patients produced using Co-60 and Ir-192 sources, (A) prescribed to Point A with identical dwell distribution, and (B) prescribed to the HR-CTV with typical clinical optimisation. (D2cc is expressed as the physical brachytherapy dose)

\begin{tabular}{|c|c|c|c|}
\hline (A) & $\begin{array}{l}\text { Mean percentage difference, } \\
\text { Co-60 to Ir-192 }\end{array}$ & SD of mean & Correlation, $p$-value \\
\hline HR-CTV V100\% & $+2.4 \%$ & 1.3 & $p<0.01$, very significant difference \\
\hline HR-CTV V150\% & $+5.9 \%$ & 3.3 & $p<0.01$, very significant difference \\
\hline HR-CTV V200\% & $+7.6 \%$ & 4.2 & $p<0.01$, very significant difference \\
\hline HR-CTV V400\% & $+22.1 \%$ & 9.4 & $p<0.01$, very significant difference \\
\hline HR-CTV D90(Gy) & $+0.4 \%$ & 2.0 & $p=0.56$, no significant difference \\
\hline Bladder D2cc & $+0.8 \%$ & 1.9 & $p=0.58$, no significant difference \\
\hline Bladder ICRU ref. point & $+0.9 \%$ & 2.0 & $p=0.11$, no significant difference \\
\hline Rectum D2cc & $+3.3 \%$ & 1.0 & $p<0.01$, very significant difference \\
\hline Rectum ICRU ref. point & $+2.2 \%$ & 2.0 & $p=0.03$, significant difference \\
\hline Sigmoid D2cc & $-0.6 \%$ & 1.9 & $p=0.69$, no significant difference \\
\hline (B) & $\begin{array}{l}\text { Mean percentage difference, } \\
\text { Co-60 to Ir-192 }\end{array}$ & SD of mean & Correlation, $p$-value \\
\hline HR-CTV V100\% & $+0.7 \%$ & 1.4 & $p=0.23$, no significant difference \\
\hline HR-CTV V150\% & $+4.4 \%$ & 4.6 & $p=0.03$, significant difference \\
\hline HR-CTV V200\% & $+7.0 \%$ & 3.0 & $p<0.01$, very significant difference \\
\hline HR-CTV V400\% & $+11.6 \%$ & 5.1 & $p<0.01$, very significant difference \\
\hline HR-CTV D90(Gy) & $-0.2 \%$ & 2.0 & $p=0.40$, no significant difference \\
\hline Bladder D2cc & $-0.4 \%$ & 2.6 & $p=0.90$, no significant difference \\
\hline Rectum D2cc & $+0.9 \%$ & 3.3 & $p=0.43$, no significant difference \\
\hline Sigmoid D2cc & $-0.3 \%$ & 3.5 & $p=0.67$, no significant difference \\
\hline
\end{tabular}

strength multiplied by the dose rate constant multiplied by time. When considering source activity, the dose is proportional to activity multiplied by air kerma rate constant, multiplied by dose rate constant, multiplied by time.

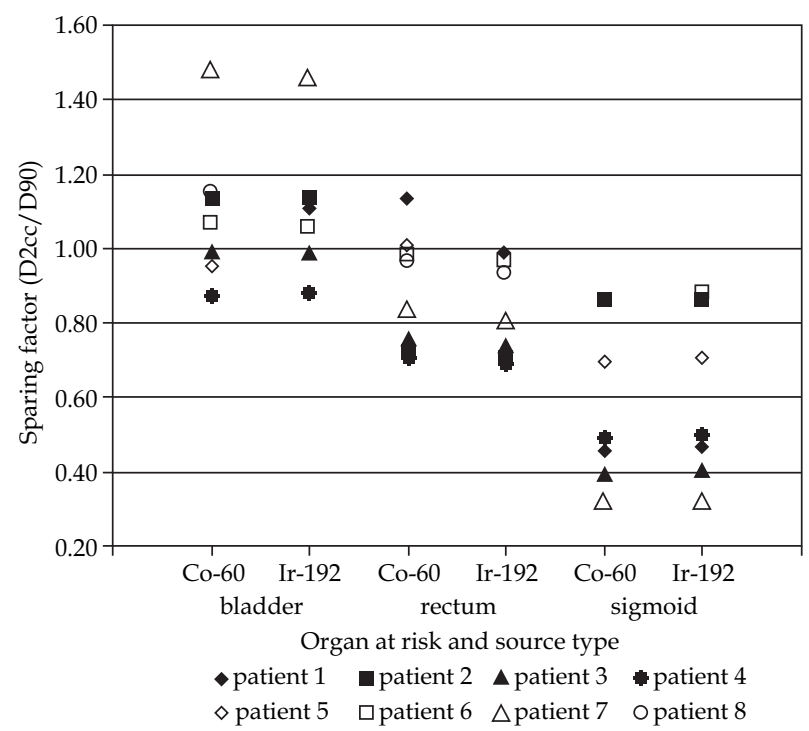

Fig. 3. Comparison of sparing factors calculated for the bladder, rectum and sigmoid in eight cervix cancer patient plans, prescribed to HR-CTV, using Co-60 and Ir-192 HDR sources. (Condition $[\mathrm{B}]$ in method)
Hence, using the data in Table 2, for HDR brachytherapy, $1 \mathrm{GBq}$ of a Co- 60 source delivers the same dose as $2.77 \mathrm{GBq}$ of an Ir-192 source (although source strength should be used, nominal activity is still often quoted and hence referred to here for completeness). Further, the required irradiation treatment time for a new Co-60 source is larger by a factor of 1.8 compared to a new Ir-192 source, based on the typical initial source activities given in Table 2 . The required irradiation time for brachytherapy treatments increases as the sources decay. Due to the different decay half-lives $\left(T_{1 / 2}\right)$ and the different initial conditions, the variation in required irradiation time with time for the two sources is shown in Fig. 4, where Co-60 source is replaced at five years and Ir-192 sources are changed every four months. For these source change frequencies, the average irradiation times per patient and similarly the total cumulative HDR irradiation time are around $25 \%$ greater for Co-60 than Ir-192. However, if the Co-60 source is replaced at four years, the average increase in treatment time compared to Ir-192 reduces to $15 \%$. There may also be a radiobiological effect that favours replacement at four years rather than five. Mazeron et al. [20] indicated that cell repair of sub-lethal damage starts at around 30 minutes after irradiation. Hence, longer treatment times may decrease the effect of radiation damage due to the initiation of repair during exposure. If a Co-60 source is changed every four years, the total treatment time never exceeds that required by an Ir-192 source, as shown in Fig. 4 and any potential effect can be mitigated. The differing energy of gamma emissions from the two 
Table 2. Selected physical properties of Ir-192 and Co-60 isotopes and HDR sources: mean energy ( $E_{\gamma}$, mean $)$ and energy range $\left(E_{\gamma}\right.$, range $)$ of gamma radiation, half-life time $\left(T_{1 / 2}\right)$, dose rate constant $(\Lambda)$, air kerma rate constant $(K)$, typical initial source activity of HDR sources $(A)$, and first tenth-value layer in concrete $\left(\mathrm{TVL}_{\text {concrete }}\right)$. $\left(\mathrm{U}=\mu \mathrm{Gy} / \mathrm{m}^{2} \mathrm{~h}\right)$. (From National Nuclear Data Centre [19])

\begin{tabular}{ccccccc} 
Isotope & $\begin{array}{c}\text { Ev, mean } \\
(\mathrm{MeV})\end{array}$ & $\begin{array}{c}\text { Ev, range } \\
(\mathrm{MeV})\end{array}$ & $\begin{array}{c}\mathrm{T}_{1 / 2} \\
\text { (months) }\end{array}$ & $\begin{array}{c}\boldsymbol{\Lambda} \\
(\mathrm{cGy} / \mathrm{hU})\end{array}$ & $\begin{array}{c}\mathrm{K} \\
(\mathrm{U} / \mathrm{GBq})\end{array}$ & $\begin{array}{c}\mathrm{A} \\
(\mathrm{GBq})\end{array}$ \\
\hline Ir-192 & 0.37 & $0.1-1.1$ & 2.4 & 1.108 & 108 & 370 \\
\hline Co-60 & 1.25 & $1.17 \& 1.33$ & 63.3 & 1.084 & 306 & 74
\end{tabular}

isotopes affects the dose distribution around the sources. This is described using a radial dose function and anisotropy function [17]. The radial dose function, which accounts for the effects of absorption and scatter in the medium, for Ir-192 source provides higher values than for Co-60 source within the region of interest in HDR brachytherapy, as shown in Fig. 2A, up to approximately $25 \mathrm{~cm}$ from the sources (the radial dose function evaluation depends on the size of the phantom used in Monte Carlo simulations, which were equivalent in the data presented). This is due to increased scatter of Ir-192 emissions compared to Co-60, due to their lower energy. However, the variation in radial dose function of the two sources is dominated by the geometric factor, which includes inverse-square law dose reduction (and effects of line source activity distribution). These physical properties therefore result in negligible differences between the two sources in dose-rate with distance from the source, perpendicular to the source axis, as shown in Fig. 2A. The variation in dose-rate with distance, along the source axis, is shown in Fig. 2B. The Co-60 and Ir-192 sources have a different magnitude of variation of dose-rate with polar angle around their line source geometries, due to relatively more self-absorption within the source for the lower energy emissions of Ir-192 compared to Co-60. This effect can be seen in Fig. 2C, with a $40 \%$ reduction in anisotropy function along the source axis ( 0 degree polar angle), at $2.0 \mathrm{~cm}$ from the source, for Ir-192 compared to Co-60.

The cost of a Co- 60 HDR source is approximately 5 times greater than the cost of an equivalent Ir-192 source (EZ BEBIG, at 2011). If typical source replacement frequencies are used, changing Co-60 source at five years and Ir-192 source every four months, an HDR brachytherapy treatment unit would require fifteen Ir-192 sources for each Co-60 source. The total cost of these sources is $275 \%$ greater for Ir-192 than for Co-60, an increase of approximately fortyfive thousand Euros over five years. The additional costs of transportation and engineer-installation of the sources increase the differential total costs by in excess of five thousand Euros over five years.

\section{Discussion}

From the analysis of eight patients' treatment plans, using a 3D image-based approach, only small differences in dose distribution were observed when using either Co-60 or Ir-192 isotopes. Variations were seen along the extension of the applicator axes, with Co-60 delivering several percent higher doses than Ir-192, due to reduced anisotropy variations compared to Ir-192, because of less self-

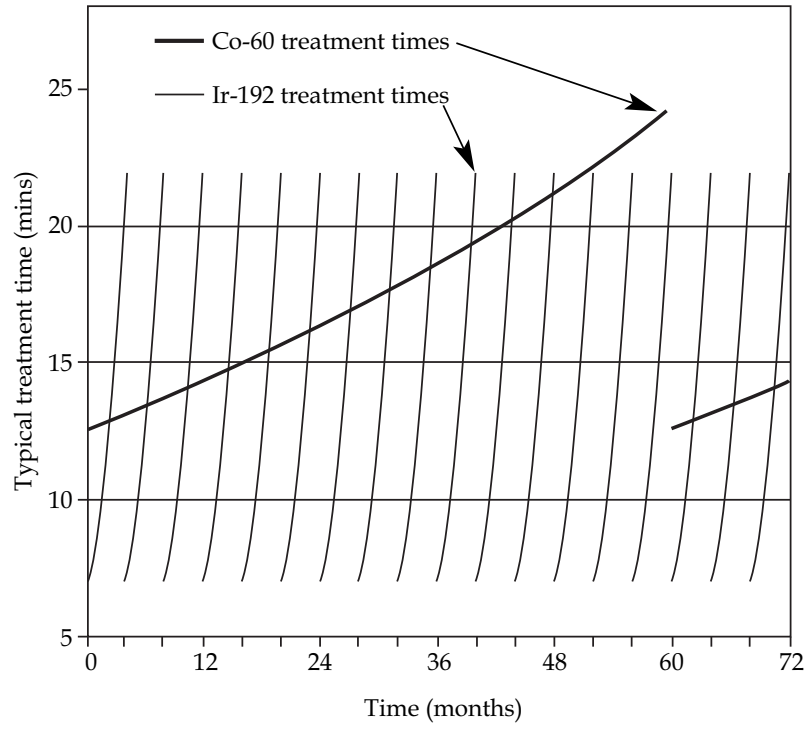

Fig. 4. Comparison of total treatment irradiation times for Co-60 and Ir-192 sources for a typical cervix HDR treatment, as a function of time over a six year period, with Co-60 source change at five years and Ir-192 source change at four months

absorption within the line source at the higher gamma emission energies. When treatment plans were prescribed to Point A and identical Manchester-based loading patterns were used, there was a small, but statistically significant increase in volume of HR-CTV covered by the prescription dose, as well as a small increase to the rectum OAR, when using Co-60 rather than Ir-192. When treatment plans were prescribed to the HR-CTV and dwell times were optimised, there was no statistically significant difference in any OAR parameter nor in the HR-CTV V100\% coverage between the two isotopes. The small, but statistically significant dose distribution differences seen when comparing identically-loaded treatment plans for Co-60 and Ir-192, which are a function of the inherent physical differences of the sources, are reduced to being not statistically significant in typical clinical treatment planning, when dwell-time optimisation is applied.

In comparing Co-60 (EZ BEBIG) and Ir-192 (Nucletron) HDR sources using a 2D image-based approach and identical loading patterns, Park et al. [11] reported an average decrease of $1.14 \pm 0.61 \%$ to the ICRU bladder reference point and an average increase of $0.83 \pm 1.48 \%$ to the ICRU rectal reference point, when using Co-60 rather than Ir- 192. 
These findings are consistent with the work presented here in terms of Point A prescribing, ICRU reference points and identical source loading. However, although of technical interest, the dose differences for identical loading and dwelltime patterns, such as that reported by Park et al. [11] and in this work under condition A are not of particular clinical significance for modern treatment planning. Of more significance are the clinical treatment plans that can be produced when optimisation of source dwells is permitted, which is a standard practice for modern brachytherapy, as reported in this work under condition B. Differences between Co-60 and Ir-192 were then reduced to $0.4 \%$ (SD = 2.6) for bladder $\mathrm{D} 2 \mathrm{cc}$ and $0.9 \%(\mathrm{SD}=3.3)$ for rectal $\mathrm{D} 2 \mathrm{cc}$, neither of which were statistically significant. The generally quoted quality metrics of HR-CTV D90Gy, V100\% and OAR D2cc do not show significant differences between the two isotopes. However, in all plans, the HR-CTV volume covered by $150 \%, 200 \%$ and $400 \%$ was significantly different, with Co-60 always delivering greater coverage. There is some debate on the clinical significance of 'over-dose' regions, indeed Prabhakar [21] has discussed several studies in which an increase in high dose regions may be a disadvantage. There was no significant difference in the bladder or rectum sparing factors, evaluated as D2cc/D90, between the two isotopes for any of the considered plans. Even when rectal doses were a few per cent higher with Co-60 compared to Ir-192, for the identical dwell-loading plans, the increase in rectal dose were consistent with increased dose to the HR$\mathrm{CTV}$; the sparing factor ratio being unchanged.

Co-60 may deliver a small increased dose to the rectum and small enhancement of the target overdose volume compared to Ir-192, depending on planning technique employed. However, the differences in volume and point doses in the treatment plans for Co-60 and Ir-192 were judged to be clinically not significant, especially in context of more significant effect of other variables that can be modified in HDR brachytherapy treatment planning, including prescribing method, loading patterns, extent of dwell-time optimisation, application of image-guidance techniques, choice of applicator, use of supplementary interstitial needles, etc. Strohmaier [8] also concluded there to be insignificant clinical differences between the two isotopes based on analyses using ICRU point doses and with 2D imagebased treatment planning. It is apparent that the inherent differences between the two isotopes, which itself lead to small differences in dose distribution, can be overcome by application of treatment planning optimisation techniques. The key physical parameters for Co-60 and Ir-192 HDR sources have been presented and analysed to deduce clinically relevant information for use in brachytherapy treatments. It is essential that this data is fully understood to mitigate the risk of any treatment error in moving from Ir-192 to Co-60. Co-60 and Ir-192 sources of identical shape and construction show practically identical dose distributions despite definite differences in the key physical characteristics of the isotopes, particularly emission spectra. This is because of the overwhelming effect of geometry in brachytherapy and small differences in radial dose distributions and anisotropy. In terms of economics, there is significant financial saving in Co-60 source replacements every five years compared to Ir-192 replacements every four months, amounting to more than fifty thousand Euros over five years (at 2011). The reduced frequency of source changes also reduces the physics calibration and quality control time, reducing the overall equipment down-time and required support time by approximately $40 \%$. However, the initial cost of environmental radiation shielding is greater for Co-60 than Ir-192, due to the higher energy emissions. The significantly longer operating life of the Co-60 source requires enhanced mechanical reliability over an equivalent Ir-192 source. This may have implications for a full-circle single-channel ring applicator due to increased mechanical wear on the source cable when traversing a small radius ring.

The irradiation time per patient, over five year use of a Co-60 source, is on average $25 \%$ greater than for Ir-192 sources. However, if patient set-up time and ancillary activities are included in the total patient treatment time, which are of course independent of source type, the percentage increase of total patient-time for Co-60 compared to Ir-192 is reduced to approximately $10 \%$.

\section{Conclusions}

Based on the 3D dosimetric analysis of patient plans considered in this study, we have shown that Co- 60 can be used as an effective substitute for the more common Ir-192 sources in HDR brachytherapy for cervix cancer, with no clinically significant differences evident in the resulting treatment plan dose distributions, especially when treatment planning dwell-time optimisation is utilised. The choice of isotope is certainly less significant than other variables in brachytherapy, including choice of prescribing method, extent of image-guidance or dose optimisation techniques.

The inherent physical differences between the sources, leads to reduced anisotropy deviations with Co-60 compared to Ir-192 at high polar angles. This causes small increases in dose deposition along the extension of the source axis with Co-60, which may increase rectal doses for identical source loading patterns compared to Ir-192. However, these differences may be eliminated with treatment plan optimisation.

Co-60 treatment plans delivered small increases in target coverage (V100) $(p<0.01)$ for standard loading and equivalent target coverage for optimised plans, compared to Ir-192. There was no statistically significant difference in D90 between the two isotopes $(p \geq 0.4)$. Increases in the overdose volume (V150 to V400) $(p=0.03$ to $<0.01)$ were observed for Co-60 compared to Ir-192 for both standard loading and optimised plans.

There are significant logistical and financial benefits of using the longer half-life Co-60 source. For the same doserate, a Co-60 source requires only $36 \%$ of the activity of an Ir-192 source. For typically supplied source strengths, the total treatment irradiation times will be on average $25 \%$ greater duration with Co-60 than Ir-192, over the lifetime of the source, however the overall patient attendance time will be approximately $10 \%$ greater due to ancillary activities independent of actual irradiation. The choice of isotope has no impact on prescription doses and fractionation schemes. 


\section{Acknowledgments}

We acknowledge Antonius Spiller of Eckert \& Ziegler BEBIG GmbH and Professor David Bradley and Professor Andrew Nisbet of University of Surrey for review of this work.

\section{References}

1. Salminen EK, Kiel K, Ibbott GS et al. International conference on advances in radiation oncology (ICARO): Outcomes of an IAEA meeting. Radiat Oncol 2011; 6: 1-9.

2. Henschke UK, Hilaris BS, Mahan GD. Remote afterloading with intracavitary applicators. Radiol 1964; 83: 344-345.

3. Nag S, Erickson B, Thomadsen B, et al. The American Brachytherapy Society recommendations for high-dose-rate brachytherapy for carcinoma of the cervix. Int J Radiat Oncol Biol Phys 2000; 48: 201-211.

4. Dale RG. Some theoretical derivations relating to the tissue dosimetry of brachytherapy nuclides, with particular reference to Iodine-125. Med Phys 1983; 10: 176-183.

5. Ntekim A, Adenipekun A, Akinlade B et al. High dose rate brachytherapy in the treatment of cervical cancer: Preliminary experience with cobalt 60 radionuclide source - a prospective study. Clin Med Insights Oncol 2010; 4: 89-94.

6. Mosalaei M, Mohammadianpanah M, Omidvari S et al. Ahmadloo N. High dose rate brachytherapy in the treatment of carcinoma of uterine cervix: twenty-year experience with cobalt after-loading systems. Int J Gynecol Cancer 2006; 16: 1101-1105.

7. Bocharova I. The history of brachytherapy in Russia: comparison of Co-60 vs, Ir-192 sources. J Contemp Brachyther 2011; 3: 48-49.

8. Strohmaier S, Zwierzchowski G. Comparison of $60 \mathrm{Co}$ and 192Ir sources in HDR brachytherapy. J Contemp Brachyther 2011; 3: 199-208

9. Venselaar JL, van der Giessen PH, Dries WJ. Measurement and calculation of the dose at large distances from brachytherapy sources: Cs-137, Ir-192 and co-60. Med Phys 1996; 23: 537-543.

10. Richter J, Baier K, Flentje M. Comparison of 60cobalt and 192iridium sources in high dose rate afterloading brachytherapy. Strahlenther Onkol 2008; 184: 187-192.

11. Park D-W, Kim YS, Park SH et al. A comparison of dose distributions of HDR intracavitary brachytherapy using different sources and treatment planning systems. Appl Radiat Isot 2009; 67: 1426-1431.

12. Chassagne D, Dutreix A, Almond P et al. Dose and volume specification for reporting intracavitary therapy in gynaecology. International Commission on Radiation Units and Measurements, ICRU Report 38, 1985.

13. Pötter R, Haie-Meder C, Van Limbergen E et al. Recommendations from gynaecological (GYN) GEC ESTRO working group (II): concepts and terms in 3D image-based treatment planning in cervix cancer brachytherapy-3D dose-volume parameters and aspects of 3D image-based anatomy, radiation physics, radiobiology. Radiother Oncol 2006; 78: 67-77.

14. Granero D, Perez-Calatayud J. Dosimetric study of a new Co-60 source used in brachytherapy. Med Phys 2007; 34: 34853488.

15. Granero D, Perez-Calatayud J. Monte Carlo study of the dose rate distributions for the Ir2.A85-2 and Ir2.A85-1 Ir-192 afterloading sources. Med Phys 2008; 35: 1280-1287.

16. Palani Selvam T, Bhola S. Technical note: EGSnrc-based dosimetric study of the BEBIG 60Co HDR brachytherapy sources. Med Phys 2010; 37: 1365-1370.

17. Rivard MJ, Coursey BM, DeWerd LA et al. Update of AAPM Task Group No. 43 report: A revised AAPM protocol for brachytherapy dose calculations. Med Phys 2004; 31: 633-674.
18. Pujades-Claumarchirant MA, Granero D, Perez-Calatayud J et al. Evaluation of interpolation methods for TG-43 dosimetric parameters based on comparison with Monte Carlo data for high-energy brachytherapy sources. J Contemp Brachyther 2010; 1 : 28-32.

19. National Nuclear Data Centre. http://www.nndc.bnl.gov/

20. Mazeron JJ, Scalliet P, Van Limbergen EV et al. Radiobiology of brachytherapy and the dose-rate effect. In: Gerbaulet A, Potter R, Mazeron JJ (eds.). The GEC ESTRO handbook of brachytherapy. ESTRO, Leuven 2002; 95-121.

21. Prabhakar R. Dose volume uniformity index: a simple tool for treatment plan evaluation in brachytherapy. J Contemp Brachyther 2010; 2 : 71-75. 